\title{
DEPOSIÇÃO E ACÚMULO DE MATÉRIA SECA E NUTRIENTES EM SERAPILHEIRA EM UM BOSQUE DE SABIÁ (Mimosa caesalpiniifolia Benth.) ${ }^{1}$
}

\author{
Rinaldo Luiz Caraciolo Ferreira ${ }^{2}$, Mario de Andrade Lira Junior ${ }^{2}$, Mallon Sampaio da Rocha ${ }^{3}$, Mércia \\ Virginia Ferreira dos Santos², Mário de Andrade Lira ${ }^{3}$ e Levy Paes Barreto²
}

\begin{abstract}
RESUMO - A quantificação da deposição de serapilheira e o acúmulo de manta orgânica são etapas importantes dos estudos de ciclagem de nutrientes. Com esse fim, objetivou-se quantificar a deposição de serapilheira e o acúmulo de manta orgânica em um povoamento de Sabiá (Mimosa caesalpiniifolia Benth.), no campo experimental pertencente à Empresa Pernambucana de Pesquisa Agropecuária - IPA, Itambé, PE. Para quantificação da serapilheira foram utilizados 20 coletores de madeira de $0,50 \times 0,50 \times 15 \mathrm{~cm}$, à distância do solo de $30 \mathrm{~cm}$, distribuídos sistematicamente na área. O material depositado foi coletado mensalmente no período de outubro/ 2000 a setembro/2001. O material colhido em cada coletor foi separado em folhas, galhos, flores, frutos e miscelânea. A manta orgânica não decomposta sobre o solo foi amostrada, ao acaso, nas proximidades da caixa coletora, obtendo-se 20 amostras mensais. De cada amostra foi retirado o material contido numa área de $30 \times 30 \mathrm{~cm}$. O material colhido foi separado em folhas, flores, frutos, miscelânea e galhos. As amostras foram levadas à estufa a $60^{\circ} \mathrm{C}$ no período de 48 horas até peso constante. A deposição mensal de serapilheira e frações folha e legume ocorreu em outubro, provavelmente devido ao início do período seco. A deposição e o estoque de serapilheira foram estimados em 7830,44 kg.ha- ${ }^{-1} \cdot$ ano $^{-1}$ e $8906.9, \mathrm{~kg}_{\text {h }} \mathrm{h}^{-1}$, respectivamente, com a fração folha dominando. A fração folha apresentou os maiores teores de nutrientes.
\end{abstract}

Palavras-chave: Ciclo de carbono, decomposição, leguminosa arbórea, ciclagem de nutrientes e tropical.

\section{LITTER DRY MASS AND NUTRIENT DEPOSITION AND ACCUMULATION IN A SABIÁ (Mimosa caesalpiniifolia Benth.) WOODLOT}

\begin{abstract}
Quantification of litter deposition and organic mantle accumulation are important steps on nutrient cycling studies. This study aimed to quantify litter deposition and organic mantle in a sabiá (Mimosa caesalpiniifolia Benth) woodlot in an experimental farm in the Brazilian northeast region. Twenty $0.50 x$ $0.50 \times 0.15 \mathrm{~m}$ wood collectors, to quantify litter deposition, were systematically distributed over the area elevated $30 \mathrm{~cm}$ above the soil. Deposited material was collected monthly from October 2000 to September 2001, and separated into leaves, twigs, flowers, pods and mixed portions. Undecomposed organic mantle was randomly sampled nearby collector boxes, to a total of 20 monthly samples, from which a $0.30 \times 0.30$ m composed sample was collected. The collected material was also hand separated into leaves, twigs, flowers,
\end{abstract}

\footnotetext{
${ }^{1}$ Recebido em 25.10.2005 e aceito para publicação em 13.09.2006.

${ }^{2}$ Universidade Federal Rural de Pernambuco, Av. D. Manoel de Medeiros, s/n Dois Irmãos, Recife, Pernambuco, 52171-900. E-mail: <rinaldo@dcfl.ufrpe.br>; <mario.lira@depa.ufrpe.br>; <mercia@dz.ufrpe.br>e <levy@ufrpe.br>.

${ }^{3}$ Graduação Engenharia Florestal, Universidade Federal Rural de Pernambuco. Av. D. Manoel de Medeiros, s/n. Dois Irmãos, Recife, Pernambuco 52171-900. mallonsampaio@ig.com.br

${ }^{4}$ Empresa Pernambucana de Pesquisa Agropecuária. Av. General San Martin, 1371, Bonji, Recife-PE. 50761-000. E-mail: <mariolira@terra.com.br>,<mallonsampaio@ig.com.br>.
} 
pods and mixed portions. All collected material was dried at $60^{\circ} \mathrm{C}$ until constant weight was achieved. Highest monthly litter deposition and leaf and pod fractions occurred in October, probably due to initiation of dry

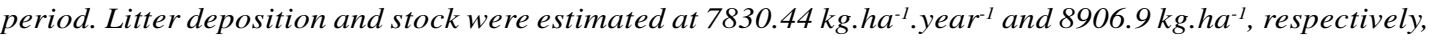
with the leaffraction dominating. Leaffraction was the richest in nutrient content.

Keywords: Carbon cycle, decomposition, legume tree, nutrient cycling and tropical.

\section{INTRODUÇÃO}

No estudo da ciclagem de nutrientes, a quantificação das reservas minerais e orgânicas e suas transferências entre compartimentos são de extrema importância para compreender e comparar os diferentes ecossistemas e suas relações com o meio (KURZATKOWSKI et al., 2004). A forma pela qual as árvores efetuam essa ciclagem, via serapilheira, é um indicativo da estratégia de adaptação das diferentes espécies às limitações nutricionais de diferentes ambientes (FERREIRA et al., 2001).

A serapilheira é a principal via de transferência no fluxo de nutrientes e fundamental para a sustentabilidade de uma floresta, pois permite que, pelo menos em parte, ocorra o retorno ao solo de uma significativa quantidade de nutrientes absorvida pelas plantas. Isso tem impacto positivo no balanço de nutrientes no sítio, diminuindo os impactos que a colheita pode causar (KETTERINGS et al., 2002; MARTIUS et al., 2004). Acamada de serapilheira, juntamente com a parte aérea e radicular das plantas, também protege o solo dos agentes erosivos e propicia condições para o reestabelecimento de suas propriedades físicas, químicas e biológicas (TILMAN et al., 2002; IKPE et al., 2003).

A dinâmica da serapilheira e de seus nutrientes, representada pela entrada via deposição e saída via decomposição/mineralização, é essencial à manutenção de florestas nativas ou plantios florestais. Em razão disso, nos últimos anos observaram-se, na literatura científica, vários trabalhos sobre ecossistemas florestais contendo serapilheira avaliadas nutricionalmente e seu fracionamento (ANDRADEetal., 2000; SCHROTHetal., 2001; FERREIRA et al., 2001; ZINN et al., 2002; DIAS et al., 2002; TOLEDO et al., 2002; KÖNIG et al., 2002a,b; SARIYILDIZ e ANDERSON, 2003; AIDAR e JOLY, 2003; ARATO et al., 2003; FIGUEIREDO FILHO etal., 2003; SCHUMACHER et al., 2003; BERTALOT et al., 2004; SCHUMACHER et al., 2004; BALIEIRO et al., 2004a).

A escolha inicial de essências florestais para formação de maciços pode refletir-se na sazonalidade, quantidade e qualidade da serapilheira produzida, alterando, conseqüentemente, as características químicas do solo e a cadeia alimentar resultante dos detritos (POGGIANI e SCHUMACHER, 2000). No Nordeste brasileiro, uma das espécies mais promissoras para implantação de florestas é o sabiá (Mimosa caesalpiniifolia Benth.), devido ao seu rápido crescimento, seu excelente valor protéico e energético como forrageira e de suas várias outras utilidades, como estacas, mourões de cerca, lenha e carvão, podendo ser estabelecida em cerca-viva e pastos arbóreos (GOMES, 1986), além da grande produção de serapilheira(COSTA,1988).

Este trabalho objetivou quantificar a deposição e acúmulo de matéria seca e de nutrientes na serapilheira em bosque de Mimosa caesalpiniifolia Benth.

\section{MATERIAL E MÉTODOS}

O experimento foi instalado no campo experimental de Itambé, pertencente à Empresa Pernambucana de Pesquisa Agropecuária - IPA e situado no Município de Itambé, PE, $7^{\circ} 41^{\prime} \mathrm{S}, 35^{\circ} 11^{\prime} \mathrm{S}$. A vegetação da área é classificada como floresta caducifólia e subcaducifólia. A precipitação média anual é de $1.300 \mathrm{~mm}$, com temperatura média anual de $26^{\circ} \mathrm{C}$ e altitude de $190 \mathrm{~m}$. O solo é predominantemente Argissolo Vermelho-Amarelo, textura franco-argilo-arenoso, com relevo plano a suaveondulado (IPA, 1994).

O bosque de sabiá foi implantado em 1990 e ocupa área de 0,30 ha. No plantio foi utilizado o espaçamento de 3,0 x 3,0 m, sendo que cada planta recebeu, na cova, $3,0 \mathrm{~kg}$ de esterco e $200 \mathrm{~g}$ de superfosfato simples por planta.

Para determinação da deposição de serapilheira foram instalados 20 coletores de madeira, com abertura de $50 \times 50 \mathrm{~cm}$, profundidade de $15 \mathrm{~cm}$ e fundo de tela de náilon com malha de $1 \mathrm{~mm}$ de abertura. Os coletores encontravam-se a uma altura de $0,3 \mathrm{~m}$ do solo e foram distribuídos sistematicamente na área. O material depositado foi coletado mensalmente no período de outubro de 2000 a setembro de 2001.

A serapilheira não decomposta sobre o solo (manta 
orgânica) foi amostrada ao acaso, obtendo-se 60 amostras, das quais foi retirada a quantidade contida numa área de $30 \times 30 \mathrm{~cm}$. O material depositado foi coletado mensalmente no período de outubro de 2000 a setembro de 2001 .

O tempo médio de residência (TMR, em anos) da serapilheira no solo, sob cada plantio, foi estimado conforme Landberg e Gower (1996), a partir da razão entre a massa média anual de manta orgânica $(\mathrm{kg}$.ha $\left.{ }^{1}\right)$ e a massa de serapilheira depositada durante o ano $\left(\mathrm{kg} \mathrm{ha}^{-1} \cdot \mathrm{ano}^{-1}\right)$.

O material colhido foi separado nas frações: folhas, galhos finos (diâmetro $£ 2 \mathrm{~cm}$ ), flores, frutos e miscelânea e foi levado à estufa a $60{ }^{\circ} \mathrm{C}$, por 48 horas, até peso constante, e triturado em um moinho de facas.

As análises para a determinação dos minerais na serapilheira por fração foram realizadas nos Laboratórios de Química Vegetal e Química do Solo da Universidade Federal de Pernambuco (UFRPE). Inicialmente, foi feita a digestão nitroperclórica; em seguida, realizaram-se análises para a determinação de potássio $(\mathrm{K})$ por fotometria e nitrogênio (N) pelo método Macro-Kheldahl, conforme Bezerra Neto et al. (1994). O teor de fósforo (P) foi determinado em espectrofotômetro de absorção atômica (MALAVOLTA et al., 1989).

\section{RESULTADOS E DISCUSSÃO}

O material formador da serapilheira é constituído principalmente por folhas, representando, em média, $70,9 \%$ dos resíduos depositados anualmente e $65,8 \%$ da acumulada (Quadro 1). Outros estudos constataram também maiores porcentagens (em média 70\%) de folhas no material depositado anualmente (ANDRADE et al., 2000; TOLEDO et al., 2002; KAUR et al., 2002; KÖNIG et al., 2002a; SCHUMACHER etal., 2003; SCHUMACHER et al., 2004).

Os frutos constituíram cerca de $15,4 \%$ dos resíduos depositados em um ano, enquanto a deposição da fração galhos constituiu em torno de 5,7\% da serapilheira, valor inferior ao encontrado por Andrade et al. (2000) para a mesma espécie e similar ao encontrado para Acacia mangium e Acacia holosericea e superior ao encontrado para Acacia mearnii por Schumacher et al. (2003). Os componentes que obtiveram menores índices foram os de miscelânea e flores com 5,5 e 2,5\%, respectivamente (Quadro 1).
Quadro 1 - Deposição anual e acúmulo de serapilheira e suas frações em bosque de sabiá, Itambé-PE

Table 1 - Annual litter deposition and accumulation and its fractions in sabiá woodlots, Itambé-Brazilian Northeast

\begin{tabular}{lcc}
\hline Fração & Deposição & Acúmulo \\
\cline { 2 - 3 } & $\mathrm{kg} / \mathrm{ha} / \mathrm{ano}$ & $\mathrm{kg} / \mathrm{ha}$ \\
\hline Folha & 5549,38 & 5858,8 \\
Galho & 446,72 & 1446,3 \\
Fruto & 1203,17 & 1527,2 \\
Flores & 196,82 & 0,0 \\
Miscelânea & 434,35 & 74,6 \\
Total & 7830,44 & 8906,9 \\
\hline
\end{tabular}

A deposição mensal da serapilheira foi estimada em 669,72 kg.ha ${ }^{-1}$, com mínima e máxima de 292,65 e $1.624,22 \mathrm{~kg} \cdot \mathrm{ha}^{-1}$, respectivamente, em junho de 2001 e novembro de 2000 (Figura 1). As diferentes épocas de máxima e mínima deposições de material formador de serapilheira observadas no sabiá são características que podem ser usadas estrategicamente para o processo de recuperação de solos (ANDRADE et al., 2000).

A relação deposição/acúmulo foi de 0,88/ano, indicando o tempo de renovação da serapilheira. Vale salientar que, quanto maior o valor dessa relação, mais rápida a velocidade de decomposição da camada de serapilheira e, conseqüentemente, a ciclagem de nutrientes no sistema.

A maior deposição mensal de serapilheira (Figura 1) e das frações folha e fruto (Figura 2), em outubro, provavelmente se deve ao início do período seco (Figura 1), corroborando a afirmativa de Gomes (1986) de que a sabiá é uma espécie que perde suas folhas com a escassez de água.

O declínio na deposição total foi mais acentuado entre os meses de outubro e novembro, devido à queda acentuada na deposição de folhas e frutos. Apesar de a correlação entre deposição e precipitação não ser significativa $(r=-0,43 ; p>0,05)$, a queda na deposição de folhas poderá ser proveniente do início de estiagem e da presença de flores e frutos (Figura 2). O declínio da fração dos frutos (Figura 2) nos meses de outubro a junho está relacionado com o final de sua produção, após o aparecimento da fração flores no mês de março, expressa em maior quantidade em abril e em declínio no mês de maio. As frações de galhos e miscelânea mantiveram-se estáveis (Figura 2), com pouca variação, exceto nos meses de abril e maio.

R. Árvore, Viçosa-MG, v.31, n.1, p.7-12, 2007 


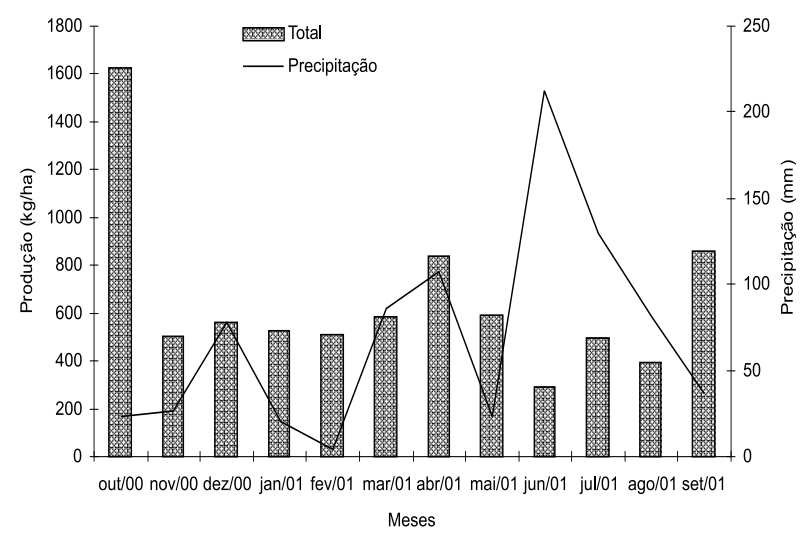

Figura 1 - Deposição mensal de serapilheira em um bosque de Mimosa caesalpiniifolia Benth., Itambé,PE.

Figure 1-Monthly litter deposition on a Mimosa caesalpiniifolia

Benth. woodlot in, Itambé, Brazilian Northeast.

A variação de deposição total ocorrida entre os meses de março e junho pode ser proveniente da estação chuvosa, em que a precipitação mensal variou de 86,2 a 212,8 mm. As menores oscilações ocorreram de novembro a março, indicando que nesse período a espécie propiciou uma proteção mais efetiva e contínua do solo.

Folhas foram os principais contribuintes de nutrientes na serapilheira depositada e acumulada (Quadro 2). A espécie depositou quantidades significativas de nutrientes, com efeitos benéficos para a melhoria da fertilidade do solo. A grande deposição de $\mathrm{N}$ provavelmente deveu-se à fixação biológica de nitrogênio. A deposição de $\mathrm{P}$ e K podem ser consideradas altas, quando comparadas com as médias observadas em florestas tropicais.
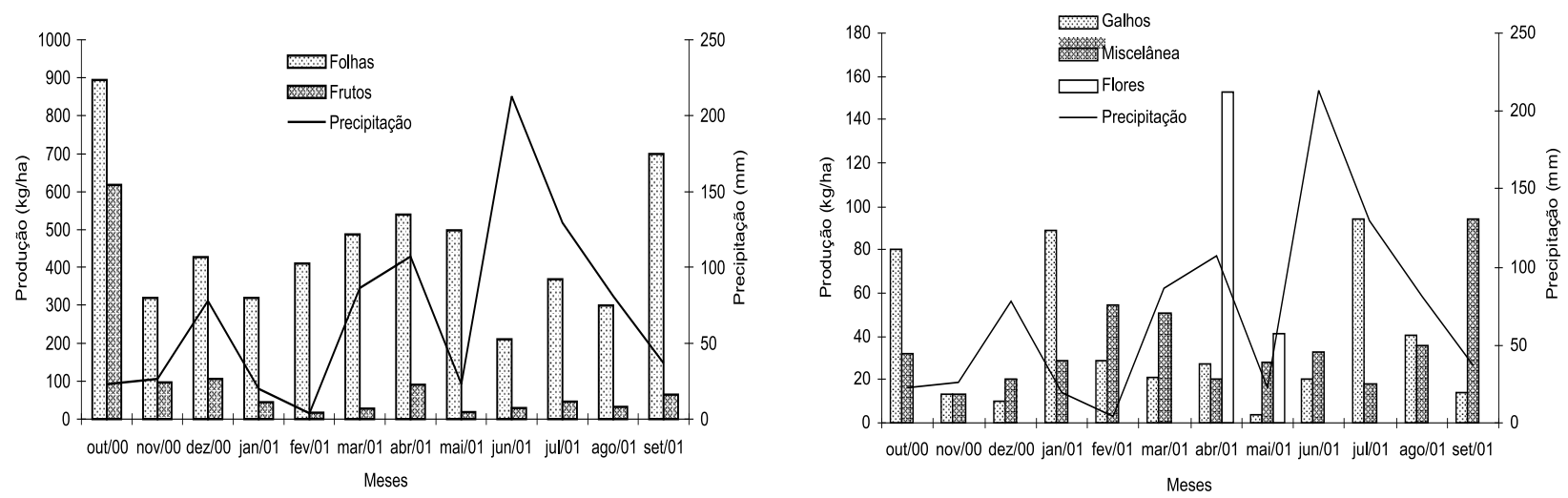

Figura 2 - Deposição mensal de serapilheira, conforme suas frações em um bosque de Mimosa caesalpiniifolia Benth., Itambé, PE. Figure 2-Monthly litter deposition according its fractions in a Mimosa caesalpiniifolia Benth. woodlot in, Itambé, Brazilian Northeast.

Quadro 2 - Deposição anual e estoque de nutrientes na serapilheira e em suas frações em bosque de Mimosa caesalpiniifolia Benth., Itambé, PE

Table 2 - Annual deposition and nutrient stock for litter and its fraction in a Mimosa caesalpiniifolia Benth woodlot. Itambé, PE

\begin{tabular}{|c|c|c|c|c|c|c|c|c|c|c|}
\hline \multirow[t]{3}{*}{ Fração } & \multicolumn{5}{|c|}{ Deposição (D) } & \multicolumn{5}{|c|}{ Acúmulo (E) } \\
\hline & $\mathrm{N}$ & $\mathrm{P}$ & $\mathrm{K}$ & $\mathrm{Ca}$ & $\mathrm{Mg}$ & $\mathrm{N}$ & $\mathrm{P}$ & $\mathrm{K}$ & $\mathrm{Ca}$ & $\mathrm{Mg}$ \\
\hline & \multicolumn{5}{|c|}{ kg.ha- ano $^{-1}$} & \multicolumn{5}{|c|}{ Kg.ha $^{-1}$} \\
\hline Folha & 87,8 & 5,1 & 29,3 & 72,5 & 17,4 & 110,3 & 5,3 & 16,3 & 83,9 & 7,0 \\
\hline Galho & 3,4 & 0,3 & 2,1 & 4,9 & 0,9 & 19,2 & 1,0 & 2,3 & 16,9 & 0,8 \\
\hline Fruto & 30,5 & 2,9 & 9,9 & 17,8 & 3,1 & 37,5 & 2,6 & 7,4 & 17,5 & 1,2 \\
\hline Flores & 29,3 & 1,4 & 7,7 & 12,5 & 3,4 & & & & & \\
\hline Miscelânea & 5,7 & 0,7 & 5,9 & 7,1 & 1,5 & 1,2 & 0,1 & 0,3 & 1,03 & 0,1 \\
\hline Total & 156,7 & 10,4 & 54,9 & 114,8 & 26,3 & 168,2 & 9,0 & 26,3 & 119,33 & 9,1 \\
\hline E/D (ano) & 1,07 & 0,87 & 0,48 & 1,04 & 0,35 & & & & & \\
\hline
\end{tabular}

R. Árvore, Viçosa-MG, v.31, n.1, p.7-12, 2007 
Sabiá acumulou maiores quantidades de $\mathrm{N}$ que os demais nutrientes avaliados (Quadro 2), corroborando o observado por outros autores (BERTALOT et al., 2004; BALIEIRO et al., 2004a,b) para outras espécies florestais. A mesma seqüência de acumulação de nutrientes $(\mathrm{N}>\mathrm{Ca}>\mathrm{K}>\mathrm{P}>\mathrm{Mg}$ ) foi observada por Balieiro et al. (2004b).

A relação estoque/deposição, que representa o tempo médio de residência dos nutrientes, indica que a serapilheira de sabiá tem alta taxa de mineralização. Os resultados obtidos neste trabalho são similares aos encontrados por Andrade et al. (2000) para a mesma espécie, Acacia mangium e Acacia holosericea e por Balieiro et al. (2004b) para a leguminosa Pseudosamanea guachapele Kunth (Harms).

\section{CONCLUSÕES}

Sabiá deposita 7.830,44 kg.ha- ${ }^{-1} \cdot \mathrm{ano}^{-1}$ de serapilheira com acúmulo de $8.906,9 \mathrm{~kg} \cdot \mathrm{ha}^{-1}$ e predominância de folhas.

A fração folha é a que apresenta maior teor de nutrientes.

\section{AGRADECIMENTOS}

Trabalho parcialmente financiado por bolsa de iniciação científica PIBIC/CNPq-UFRPE para o terceiro autor e bolsas de produtividade do CNPq para os primeiro, quarto e quinto autores.

\section{REFERÊNCIAS}

AIDAR, M. P. M.; JOLY, C. A. Dinâmica da produção e decomposição da serapilheira de araribá (Centrolobium tomentosum Guill. ex Benth. - Fabaceae) em uma mata ciliar, Rio JacaréPepira, São Paulo. Revista Brasileira de Botânica, v.26, p.193-202, 2003.

ANDRADE, A. G.; COSTA, G. S.; FARIA, S. M. Deposição e decomposição da serrapilheira em povoamentos de Mimosa caesalpiniifolia, Acacia mangium e Acacia holosericea com quatro anos de idade em planossolo. Revista Brasileira de Ciência do Solo, v.24, p.777-785, 2000.

ARATO, H. D.; MARTINS, S. V.; FERRARI, S. H. S. Produção e decomposição de serapilheira em um sistema agroflorestal implantado para recuperação de área degradada em Viçosa-MG. Revista Árvore, v.27, p.715-721, 2003.
BALIEIRO, F. C. et al. Acúmulo de nutrientes na parte aérea, na serapilheira acumulada sobre o solo e decomposição de filódios de Acacia mangium Willd. Ciência Florestal, v.14, p.59-65, 2004a.

BALIEIRO, F. C. et al. Dinâmica da serapilheira e transferência de nitrogênio ao solo, em plantios de Pseudosamanea guachapele e Eucalyptus grandis. Pesquisa Agropecuária Brasileira, v.39, p.597-601, 2004b.

BERTALOT, M. J. A. et al. Retorno de nutrientes ao solo via deposição de serapilheira de quatro espécies leguminosas arbóreas na região de Botucatu-SP. Scientia Forestalis, v.65, p.219-227, 2004.

BEZERRA NETO, E.; ANDRADE, A. G.; BARRETO, L. P. Analises químicas de tecidos e produtos vegetais. Recife: Universidade Federal Rural de Pernambuco, 1994.

COSTA, M. G. O sabiá. Mossoró: Escola Superior de Agricultura de Mossoró, 1988.514 - Série B

DIAS, H. C. T. et al. Variação temporal de nutrientes na serapilheira de um fragmento de floresta estacional semidecidual montana em Lavras-MG. Cerne, v.8, p.1-16, 2002.

FERREIRA, C. A. et al. Deposição de material orgânico e nutrientes em plantios de E. grandis em diferentes regimes de adubação. Boletim de Pesquisa Florestal, v.43, p.75-86, 2001.

FIGUEIREDO FILHO, A. et al. Avaliação estacional da deposição da serapilheira em uma floresta ombrófila mista localizada no sul do estado do Paraná. Ciência Florestal, v.13, p.11-18, 2003.

GOMES, R. P. Forragens fartas na seca. São Paulo: Nobel, 1986. 233p.

IKPE, F. N.; OWOEYE, L. G.; GICHURU, M. P. Nutrient recycling potential of Tephrosia candida in cropping systems of southeastern Nigeria.

Nutrient Cycling in Agroecosystems, v.67, p.129-136, 2003.

R. Árvore, Viçosa-MG, v.31, n.1, p.7-12, 2007 
IPA. Banco de dados agrometeorológicos. Recife: 1994.100p.

KAUR, B.; GUPTA, S. R.; SINGH, G. Carbon storage and nitrogen cycling in silvopastoral systems on a sodic soil in northwestern India. Agroforestry Systems, v.54, p.21-29, 2002.

KETTERINGS, Q. M.; VANNOORDWIJK, M.; BIGHAM, J. M. Soil phosphorus availability after slash-and-burn fires of different intensities in rubber agroforests in Sumatra, Indonesia.

Agriculture, Ecosystems and Environments, v.92, p.37-48, 2002.

KÖNIG, F. G. et al. Devolução de nutrientes via serapilheira em um fragmento de Floresta Estacional Decidual no município de Santa MariaRS. Brasil Florestal, v.74, p.45-52, 2002 a.

KÖNIG, F. G. et al. Avaliação da sazonalidade da produção de serapilheira numa Floresta Estacional Decidual no município de Santa Maria-RS.

Revista Árvore, v.26, p.426-435, 2002 b.

KURZATKOWSKI, D. et al. Litter decomposition, microbial biomass and activity of soil organisms in three agroforestry sites in central Amazonia. Nutrient Cycling in Agroecosystems, v.69, p.257-267, 2004.

LANDBERG, J. J.; GOWER, S. T. Applications of physiological ecology to forest management. San Diego: Academic Publishers, 1996. p.89-124.

MALAVOLTA, E.; VITTI, G. C.; OLIVEIRA, S. A. Avaliação do estado nutricional das plantas: princípios e aplicações. Piracicaba: Potafós, 1989.201p.

MARTIUS, C. et al. Litter fall, litter stocks and decomposition rates in rainforest and agroforestry sites in central Amazonia. Nutrient Cycling in Agroecosystems, v.68, p.137-154, 2004.
POGGIANI, F.; SCHUMACHER, M. V.

Ciclagem de nutrientes em florestas nativas. In: GONÇALVES, J. L. M.;

BENEDETTI, V. Nutrição e fertilização florestal. Piracicaba: IPEF, 2000. p.287-308.

SARIYILDIZ, T.; ANDERSON, J. M. Interactions between litter quality, decomposition and soil fertility: a laboratory study. Soil Biology and Biochemistry, v.35, p.391-399, 2003.

SCHROTH, G. et al. Nutrient fluxes in rainfall, throughfall and stemflow in tree-based land use systems and spontaneous tree vegetation of central Amazonia. Agriculture Ecosystems \& Environment, v.87, p.37-49, 2001.

SCHUMACHER, M. V. et al. Produção de serapilheira em uma floresta de Araucaria angustifolia (Bertol) Kuntze no município de Pinhal Grande-RS. Revista Árvore, v.28, p.29-37, 2004.

SCHUMACHER, M. V. et al. Retorno de nutrientes via deposição de serapilheira em um povoamento de Acácia-Negra (Acacia mearnsii De Wild) no Estado do Rio Grande do Sul. Revista Árvore, v.27, p.791-798, 2003.

TILMAN, D. et al. Agricultural sustainability and intensive production practices. Nature, v.418, p.671-677, 2002.

TOLEDO, L. O.; PEREIRA, M. G.; MENEZES, C. E. G. Produção de serapilheira e transferência de nutrientes em florestas secundárias localizadas na região de Pinheiral, RJ. Ciência Florestal, v.12, p.9-16, 2002.

ZINN, Y. L.; RESCK, D. V. S.; SILVA, J. E. Soil organic carbon as affected by afforestation with Eucalyptus and Pinus in the Cerrado region of Brazil. Forest Ecology and Management, v.166, p.285-294, 2002. 